MATEC Web of Conferences 47, 03017 (2016)

DOI: $10.1051 /$ matecconf/20164703017

(C) Owned by the authors, published by EDP Sciences, 2016

\title{
Estimate of Small Stiffness and Damping Ratio in Residual Soil Using Spectral Analysis of Surface Wave Method
}

\author{
Nor Faizah Bawadi ${ }^{1,2, a}$, Khairul Anuar Mohd Nayan², Mohd Raihan Taha ${ }^{2}$ and Noor Adila Omar ${ }^{2}$ \\ ${ }^{1}$ School of Engineering and Environment, Universiti Malaysia Perlis, 02600 Arau, Perlis, Malaysia \\ ${ }^{2}$ Department of Civil and Structural Engineering, Universiti Kebangsaan Malaysia, 43600 Bangi, Selangor, Malaysia
}

\begin{abstract}
Research in the important parameters for modeling the dynamic behavior of soils has led to rapid development of the small strain stiffness and damping ratio for use in the seismic method. It is because, the experimental determination of the damping ratio is problematic, especially for hard soils sample. Many researchers have proved that the surface wave method is a reliable tool to determine shear wave velocity and damping ratio profiles at a site with very small strains level. Surface wave methods based on Rayleigh waves propagation and the resulting attenuation curve can become erroneous when higher modes contribute to the soil's response. In this study, two approaches has been used to determine the shear strain amplitude and damping ratio of residual soils at small strain level using Spectral Analysis of Surface Wave (SASW) method. One is to derive shear strain amplitude from the frequency-response curve and the other is to derive damping ratio from travel-time data. Then, the results are compared to the conventional method.
\end{abstract}

\section{Introduction}

In many geotechnical engineering applications, the level of strains in the soil is small [9, 11], especially when dealing with normal functionality of the structures. Within the range of small strain (10-4\% or less), [17], the mechanical behavior of the ground can be properly modeled by means of viscoelasticity [3]. Depending on the history of loading, soil may compact or dilate, its stiffness may depend on the magnitude of stress levels, soil deformations are time-dependent, etc. In fact, soil behavior is considered to be truly elastic in the range of small strain.

Specifically, the shear modulus and damping ratio profiles at a site can be determined by means of Rayleigh waves propagation [14], by assuming a layered half-space with horizontal, mono-phase, homogeneous and isotropic layers. Rayleigh waves propagate on the free surface of a medium and during propagation they are subjected to dispersion and attenuation. Generally, viscoelasticity dispersion and attenuation phenomena are correlated [8], so that shear modulus and damping ratio are coupled dynamic properties of the soil. Nevertheless, at very small deformations uncoupling dispersion and attenuation phenomena may represent a good approximation of soil behavior.

\footnotetext{
${ }^{a}$ Corresponding author : faizahbawadi@yahoo.com
} 


\section{Basic Theory of Seismic Method}

In current practice of geotechnical testing, the measurement of low-strain dynamic properties of soils can be performed with a variety of techniques using in-situ or laboratory tests. Surface wave measurements has been used to investigate the attenuation and damping ratio of a layered soil deposit [13]. In their studies, an attenuation curve was constructed from the observed spatial attenuation of Rayleigh wave amplitudes and then was inverted to obtain the material shear damping ratio. The strain levels can be categorized into three groups: the very small strain level, where the stiffness modulus is constant in the elastic range; the small strain level, where the stiffness modulus varies non-linearly with the strain; and large strain level, where the soil is close to failure and the soil stiffness is relatively small $[2,5,10,15]$. The significance of small strain non-linear behavior of soils in deep excavations also was examined [7].

\subsection{Strain amplitudes of soil deposit}

A review of literature revealed that there are a number of factors affecting the estimation of soil stiffness at small strain level. These factors are not only related to natural and soil inherent structures but also to its geological history. Initial shear modulus can be defined as shear modulus at infinitely small strain amplitude. It is therefore necessary to obtain strain amplitude in soil deposits when the SASW test is to be conducted. The strain amplitude, $\gamma$ is defined as the ratio of peak particle velocity, $v$, to shear wave velocity, $\mathrm{V}_{\mathrm{s}}$ :

$$
\gamma=\left(\mathrm{v} / \mathrm{V}_{\mathrm{s}}\right)
$$

At small strain conditions, particle motion resulting from propagation of shear waves is nondestructive. As $\gamma$ increases, the shear modulus will decrease from the maximum small strain value, $\mathrm{G}_{0}$. In-situ test have commonly been assumed to be small strain and the measurement of $\mathrm{V}_{\mathrm{s}}$ will be directly related to the G0 [16]. The appropriate strain must be known, as well as the appropriate modulus reduction with increasing strain.

\subsection{Damping ratio and shear stress of soil}

The $\mathrm{G}_{0}$ is an important soil parameter related to the predictions of the ground movements and field data interpretations. The increase of the internal disorder in the material and hence increase of the dissipative properties [9], in terms of material damping. In soil dynamics and earthquake engineering, the $G_{\max }$ and the damping, $D$ ratio are important parameters in soil characterization. Through the relationship of damping and shear strain $[1,12]$, the characteristic of normal or shear strain can be determined by using following equation:

$$
\mathrm{D}=\left(\mathrm{D}_{\max } / 1+\ln \left(1+(\mathrm{e}-1)\left(\gamma_{\mathrm{r}} / 2 \gamma\right)\right)\right)
$$

where $\mathrm{D}_{\max }$ is maximum damping, $33 \%$, D is damping at corresponding strain level and $\gamma$ is the shear strain at measured damping. An elastic model is not able to describe energy dissipation and hence a viscoelastic model must be used. The dissipative phenomena take place at very low cyclic strain in soils and must be taken into consideration. Soil dynamics tests under cyclic loading, both in normal triaxial or shear test have shown common hysteretic behavior. The skeleton or the backbone curve of the hysteresis is known to follow the form of curve [4]. This curve can be expressed in the form of hyperbolic stress-strain soil model which the shear stress, $\tau$ of soil can be defined using the following equation:

$$
\tau=\left(\mathrm{G}_{0} \gamma /\left(1+\left(\gamma / \gamma_{\mathrm{r}}\right)\right)\right)
$$


where $\mathrm{G}_{0}$ is shear modulus at small strain (less than about $10^{-4} \%$ ), $\gamma$ is shear strain under loading and $\gamma_{\mathrm{r}}$ is the characteristics of shear strain.

\section{Research Method}

In this study, determination of importance parameters of residuals soils was performed by using the following procedure: (1) measuring $\mathrm{V}_{\mathrm{s}}$ at the field and (2) estimating the variation of $\mathrm{G}_{0}$ and $\mathrm{D}$ of soil corresponding $\gamma$ level, based on interpretation from WinSASW 3.2.12 software.

\subsection{SASW field testing}

The field measurements were conducted using a seismograph and three vertical geophones having a natural frequency of $1 \mathrm{~Hz}$ and geophone calibration factor is $400 \mathrm{~V} / \mathrm{m} / \mathrm{s}$. The test site was a residual soil with meta-sedimentary origin where 27 stories commercial building is to be build located at Damansara, Selangor. The configuration of field measurements was set up using Common Array Profiling (CAP) [6]. Four different seismic impact sources were used to generate energy over a broad frequency range. The location of Spectral Analysis and Surface Wave (SASW) test performed at the nearest point of Standard Penetration Test (SPT) and Instrumented Pile Load (IPLT) has shown in Figure 1.

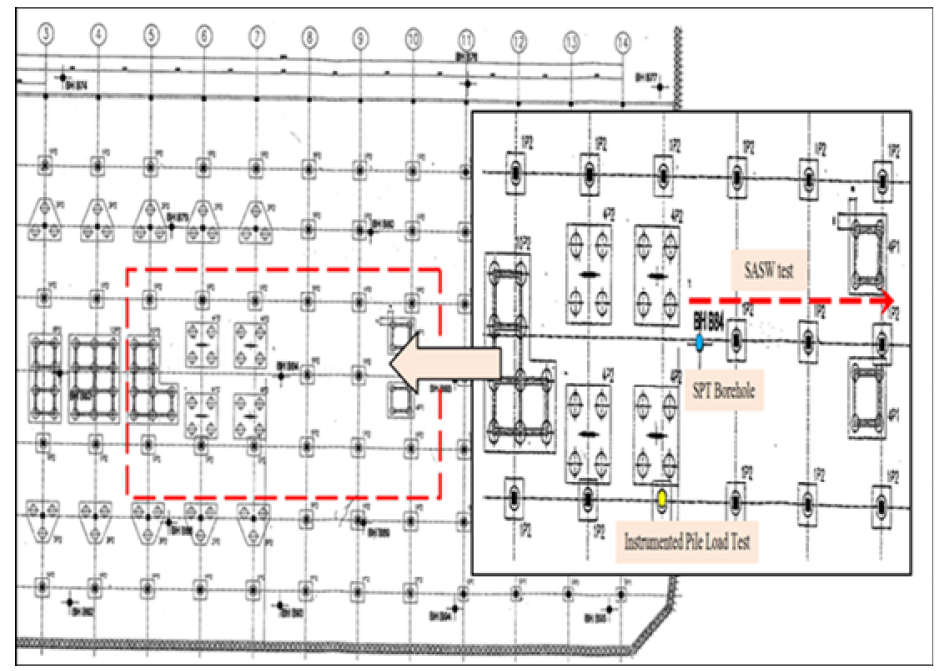

Figure 1. Site map with marked testing locations (N.T.S drawing).

\subsection{Data analysis and interpretation}

The SASW data was obtained using the National Instrument USB6289 data acquisition system with the WinSASW 3.2.12 and analysis software to determine $\mathrm{V}_{\mathrm{s}}$. This paper summarized a procedure for estimating the shear strain amplitude and damping ratio of soils at small-strain level from SASW method. The shear strain amplitude estimated from travel-time data as shown in Figure 2(a) and damping of soil measured from frequency-response curve as shown in Figure 2(b). In addition, the result obtained at a site with the two approaches are compared to the conventional method. 


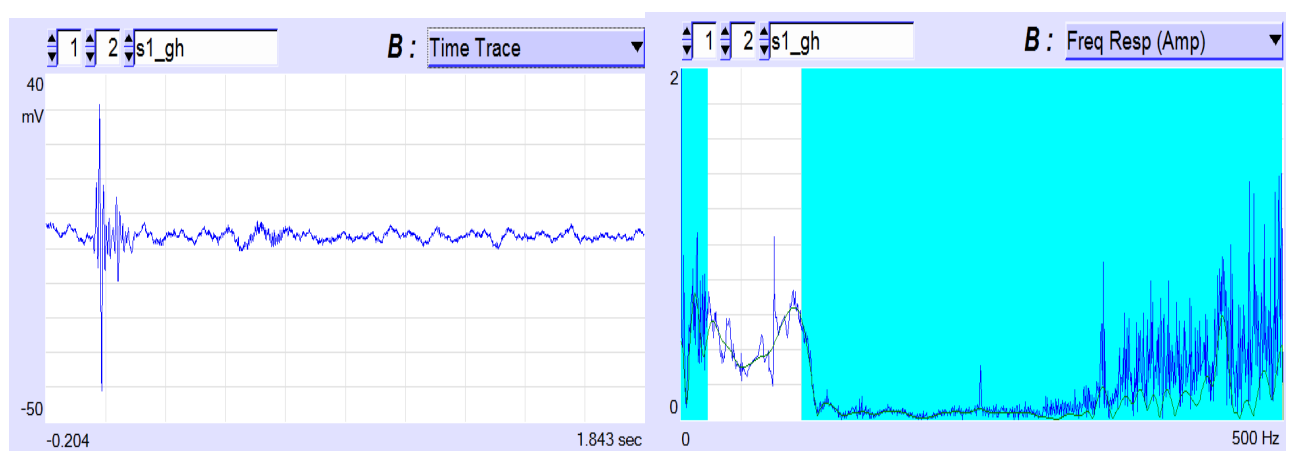

Figure 2. Data analysis for (a) travel-time curve and (b) frequency-response curve in determining strain amplitude and damping of soil.

\section{Results and Discussion}

The SPT and SASW test results identified numerous soil layers at the site indicate over 20.30 meters of medium dense silty sand, stiff sandy clay and silt which is a residual soil and has underlain by weathered rock deposit of granite as provided in Figure 3. Direct comparison of the shear wave velocity, $\mathrm{V}_{\mathrm{s}}$ and normalized shear wave velocity, $\mathrm{V}_{\mathrm{s} 1}$ profiles is illustrated in Figure $4(\mathrm{a})$. The $\mathrm{V}_{\mathrm{s}}$ profile is roughly divided into three sections, $V_{\mathrm{s}}$ is about $218 \mathrm{~m} / \mathrm{s}, 257 \mathrm{~m} / \mathrm{s}$ and $516 \mathrm{~m} / \mathrm{s}$ at $0 \sim 6.45 \mathrm{~m}$, $6.45 \sim 7.60 \mathrm{~m}$ and below $7.6 \mathrm{~m}$ which encountered the rock formation respectively. The $\mathrm{V}_{\mathrm{s} 1}$ performed after consider the overburden pressure of the soil layer.



Figure 3. The soil profile of the test site.

The dynamic strain level caused by the shear waves can be estimated based on attenuation response on the soil by conducting SASW testing. In this study, the peak particle velocity can be defined by the interpretation of travel-time data that obtained from WinSASW software. Furthermore, the results of shear strain amplitudes for all sources can be determined using Equation (1) as presented in Figure 4(b). Meanwhile, in order to measure the damping from the signal spectrum recorded from SASW test, the half-power bandwidth method has been used for the interpretation of frequencyresponse curve using Equation (2). The results of damping based on this interpretation has shown in Figure 4(c). 



Figure 4. Profile of (a) shear wave velocity, (b) strain amplitude and (c) damping for the test site using SASW method.

In general, prediction on characteristic of strain amplitudes and damping in SASW method will decreases with depth of soil. In this study, analysis of the performed SASW field data shows that the strain amplitudes caused by several assorted hammer as seismic source are generally less than $10^{-4} \%$. Summary of the shear wave velocity, shear strain amplitudes and damping as tabulated in the Table 1 below.

Table 1. Summary of the result for the test site.

\begin{tabular}{|c|c|c|c|c|c|c|c|}
\hline \multirow[b]{2}{*}{ Layer } & \multirow[b]{2}{*}{$\begin{array}{l}\text { Depth, } \\
\text { z (m) }\end{array}$} & \multirow{2}{*}{$\begin{array}{c}\text { Shear } \\
\text { Wave } \\
\text { Velocity, } \\
\mathbf{V}_{\mathbf{s}}(\mathbf{m} / \mathbf{s})\end{array}$} & \multirow{2}{*}{$\begin{array}{c}\text { Shear } \\
\text { Modulus, } \\
\mathbf{G}_{0} \\
\left(\mathbf{k N} / \mathbf{m}^{2}\right)\end{array}$} & \multicolumn{2}{|c|}{$\begin{array}{c}\text { Shear Strain } \\
\text { Amplitudes, } \gamma\end{array}$} & \multirow[b]{2}{*}{$\begin{array}{l}\text { Damping, } \\
\text { D (\%) }\end{array}$} & \multirow{2}{*}{$\begin{array}{c}\text { Shear } \\
\text { Stress } \\
\text { of } \\
\text { Soil, } \tau \\
(\mathrm{kN})\end{array}$} \\
\hline & & & & $\begin{array}{c}\text { SASW } \\
\text { Test } \\
\left(\mathbf{x 1 0}^{-6}\right)\end{array}$ & $\begin{array}{c}\text { IPLT } \\
\text { Test } \\
\left(\mathbf{x 1 0}^{-4}\right)\end{array}$ & & \\
\hline 1 & 0 & 64.48 & 6494.2 & 3.5 & 4.6 & 9.8 & 242.46 \\
\hline 2 & 0.28 & 83.33 & 5373.9 & 2.4 & 4.6 & 8.2 & 275.29 \\
\hline 3 & 1.08 & 74.68 & 3368.1 & 2.06 & 4.52 & 9.4 & 88.15 \\
\hline 4 & 1.63 & 197.09 & 21516.7 & 1.56 & 4.46 & 10.8 & 258.84 \\
\hline 5 & 2.34 & 93.04 & 3536.4 & 1.29 & 4.41 & 10.8 & 35.31 \\
\hline 6 & 3.02 & 237.99 & 16672.2 & 1.10 & 4.3 & 8.7 & 313.93 \\
\hline 7 & 6.45 & 95.46 & 2019.6 & 0.78 & 3.77 & 9.9 & 17.02 \\
\hline 8 & 7.6 & 212.22 & 11323.9 & 0.54 & 3.36 & 9.5 & 66.69 \\
\hline 9 & 8.57 & 647.32 & 71235.8 & 0.34 & 2.7 & 9.5 & 261.96 \\
\hline
\end{tabular}

From this study, the result shows that as shear strain increases, the shear modulus will decreased. The appropriate shear strain must be known, as well as the appropriate modulus reduction with increasing strain. Shear stress, $\tau$ of the soil have been determined using Equation (3) and the result has been tabulated in Table 1 above. The shear stress for a different types of soil affected by the dynamics properties of soils such as shear wave velocity, shear modulus, strain amplitudes and damping.

\section{Conclusions}

As a conclusion, it can be summarized that the effects of shear strain amplitudes on shear modulus and damping can significantly influenced the predicted shear stress of soil. Thus, shear strain amplitudes should be considered when selecting shear modulus and damping for geotechnical engineering design. 
It is also shown that the characterization of the physical properties of the soil structure in terms of the shear strain amplitudes and the damping of soil layer can be satisfactory obtained using frequencyresponse and travel-time curve from SASW data analysis. In addition, this method has the advantage of being fast, practical and non-destructive tool for engineers in measuring shear modulus and damping at very small strain conditions compared to the conventional method. Implication of the results and future research directions are also presented.

\section{References}

[1] C.P. Abbiss, Calculation of elasticities and settlements for long periods of time and high strains from seismic measurements. Geotechnique, 33(4), 397-405, (1983).

[2] J. H. Atkinson and G. Sallfors, Experimental determination of stress-strain-time characteristics in laboratory and in situ tests, Proc. of the 10th European Conference on Soil Mechanics and Foundation Engineering, Florence, Italy, 915-956, (1991).

[3] R.M. Christensen, Theory of Viscoelasticity - An Introduction, Ed. Academic Press, 245, (1971).

[4] B.O. Hardin and V.P. Drnevich, Shear modulus and damping in soils, J. of the Soil Mechanics and Foundation Division ASCE, 98(SM7), 667-692, (1972).

[5] K. Ishihara, Soil Behavior in Earthquake Geotechnics, Oxford University Press, New York, (1996).

[6] Joh Sung-Ho, Jang Dae-Woo, Kang Tae-Ho and Lee Il-Wha, Evaluation of stiffness profile for a subgrade cross-section by the CAP(Common-Array-Profiling)-SASW technique, J. of the Korean Geotechnical Society, Korean Geotechnical Society, 21(4), 71-81, (2005).

[7] G.T.C Kung, C.Y. Ou and C.H. Juang, Modelling small-strain behavior of Taipei clays for finite element analysis of braced excavations, Computer and Geotechnics, 36(1), 304-319, (2009)

[8] C.G. Lai, Simultaneous inversion of Rayleigh phase velocity and attenuation for near-surface site characterization, PhD Dissertation, Georgia Institute of Technology, Atlanta, (1998).

[9] R. Lancellotta, Geotechnical Engineering, second ed., Taylor and Francis, New York, (2009).

[10] R.J .Mair, Developments in geotechnical engineering research: application to tunnels and deep excavations, Proc. of the Institution of Civil Engineers-Civil Engineering, Unwin Memorial Lecture 1992, 97(1), 27-41, (1993).

[11] C. Mancuso, R. Vassallo and A. d'Onofrio, Small strain behavior of a silty sand in controlledsuction resonant column-torsional shear tests, Canadian Geotechnical J., 39(1), 22-31, (2002).

[12] K.A.M. Nayan, M.R. Taha, N.A. Omar, N.F. Bawadi, S.H. Joh and M.N. Omar, Determination of ultimate pile bearing capacity from a seismic method of shear wave velocity in comparison with conventional methods, J. of Technology (Sciences and Engineering), 74(3), 99-102, (2015).

[13] G.J. Rix, C.G. Lai and A.W. Spang Jr., In-situ measurement of damping ratio using surface waves, J. of Geotechnical and Geo-environmental Engineering, Proc. of the ASCE, 126(5), 472480, (2000).

[14] G.J. Rix, C.G. Lai and S. Foti, Simultaneous measurement of surface wave dispersion and attenuation curves, Geotechnical Testing J., 24(4), 350 - 358, (2001).

[15] A. Sawangsuriya, P. J. Bosscher and T. B. Edil, Alternative testing techniques for modulus of pavement bases and subgrades, Proc. of the 13th Annual Great Lakes Geotechnical and Geoenvironmental Engineering Conference, Geotechnical Applications for Transportation Infrastructure, ASCE, Geotechnical Practice Publication, 108-121, (2005).

[16] S. S. Tezcan, Z. Ozdemir and A. Keceli, Allowable bearing capacity of shallow foundations based on shear wave velocity, J. of Geotechnical and Geological Engineering, 24, 203-218, (2006).

[17] M. Vucetic, Cyclic threshold shear strains in soils, J. of Geotechnical Engineering, 120(12), 2208-2228, (1994). 